\title{
INYECCIÓN ULTRASÓNICA DE GASOLINA
}

\section{ULTRASONIC GASOLINE INJECTION}

\author{
Ing. Rubén Carlos Esquivel Hernández ", PhD. Milton Carlos Álvarez Sánchez ${ }^{*}$ \\ PhD. William Vicente ${ }^{*}$ \\ " UNAM, Instituto de Ingeniería, CIPIA. \\ Circuito Escolar s/n, Ciudad Universitaria, Coyoacán, CDMX, 04510, México. \\ Teléfono +52 (55) 56233600 ext. 8762. Fax +52 (55) 56162164. \\ E-mail: $\{$ REsquivelH, MAlvarezS, WVicenteR $\} @$ iingen.unam.mx.
}

\begin{abstract}
Resumen: La atomización ultrasónica de combustibles produce mezclas de partículas más finas y uniformes, por lo que el presente trabajo estudia los cambios en las emisiones de un motor de gasolina de cuatro tiempos equipado con un atomizador ultrasónico de combustible con respecto de su carburador original. El atomizador se diseñó para satisfacer la demanda de consumo de gasolina del motor y un sistema de muestreo se implementó para realizar las mediciones de las emisiones. El atomizador ultrasónico disminuyó las emisiones de $\mathrm{CO}$ y $\mathrm{CO} 2$ pero elevó los HC.
\end{abstract}

Palabras clave: Ultrasonido, atomización de combustible, atomización ultrasónica, emisiones contaminantes.

\begin{abstract}
The ultrasonic atomization of fuels produces mixtures of finer and uniform particles. This paper studies the changes in emissions of a four-stroke gasoline engine equipped with an ultrasonic fuel atomizer with respect to its original carburetor. The atomizer was designed to meet the fuel consumption demand of the engine and a sampling system was implemented to perform the measurements. The ultrasonic atomizer reduced $\mathrm{CO} 2$ and $\mathrm{CO}$ emissions but increased the $\mathrm{HC}$ ones.
\end{abstract}

Keywords: Ultrasound, fuel atomization, ultrasonic fuel atomization, pollutant emissions.

\section{INTRODUCCIÓN}

La principal forma de producir energía a nivel mundial continúa siendo mediante la ignición de combustibles fósiles (International Energy Agency, 2017). Debido a la contaminación emitida por este proceso, en todo el mundo se están adoptando normativas que reducen las emisiones de gases de efecto invernadero y mantienen estándares de calidad del aire para cuidar la salud pública.

La industria del transporte trabaja principalmente con motores de combustión interna (BP, 2018). En el 2013 97\% de los automóviles en circulación en el mundo eran de combustión, mientras $2.8 \%$ eran híbridos y $0.2 \%$ eléctricos. Los pronósticos más optimistas estiman que para el 2025 podría haber 70 millones de vehículos eléctricos en circulación en el mundo. No obstante, tal incremento representará menos del $10 \%$ de los vehículos en circulación, no impactando significativamente en la producción de gases de efecto invernadero $y$ emisiones contaminantes (Winton, 2016). La dominación de la combustión en este sector se regula con legislaciones que controlan las emisiones y que representan nuevos retos para la industria del transporte. 
Los motores de combustión interna queman una mezcla de aire-combustible. El tamaño y la concentración de las partículas de combustible afectan la velocidad de combustión, la cantidad de combustible quemado, la temperatura del motor y la auto-ignición de la misma. Tales factores impactan el desempeño del vehículo: eficiencia, potencia, consumo de combustible, operación del convertidor catalítico, y como consecuencia, las emisiones resultantes.

\section{Atomización ultrasónica}

Un fenómeno causado por la aplicación de ondas ultrasónicas de alta intensidad en líquidos es su atomización. La producción de neblina es proporcional a la potencia aplicada, mientras que el diámetro de las partículas es función de la frecuencia y las propiedades del fluido.

Respecto a los sistemas de pulverización de combustible modernos, un atomizador ultrasónico puede producir una neblina de partículas con un diámetro más pequeño y una distribución más estrecha (Tanyeri y Öner, 2014). Respecto a la inyección, la atomización ultrasónica no requiere de presurizar el combustible para pulverizarlo, evitando pérdidas del trabajo indicado por la bomba de alta presión. La combustión se beneficia de una evaporación más rápida y completa y de una mezcla homogénea de aire-combustible que reduce las emisiones contaminantes y el consumo de combustible. El arranque en frío del motor se beneficia de un aumento de temperatura más rápido.

La posibilidad de utilizar ultrasonido como medio de pulverización de combustible ha sido estudiada tanto en trabajos científicos como en publicaciones informales en línea (Furu, 2012).

Namiyama et al. (1989) desarrollaron un inyector de gasolina ultrasónico y obtuvieron una mayor eficiencia, mejor aceleración y menores emisiones contaminantes de monóxido de carbono e hidrocarburos, pero un aumento en los óxidos de nitrógeno; ellos realizaron las pruebas en un motor de motocicleta de un cilindro, en un automóvil de cuatro cilindros de inyección monopunto y en un automóvil de cuatro cilindros de inyección multipunto.

Kumar et al. (2013) compararon el desempeño de un atomizador ultrasónico en un motor de motocicleta con respecto del carburador original; observando un menor consumo de combustible, mayor eficiencia, menores emisiones de monóxido de carbono e hidrocarburos, pero un aumento considerable en los óxidos de nitrógeno y una capacidad de operar con relaciones airecombustible de hasta 25:1.

Balasubramanian et al. (2015) compararon el desempeño de un motor de dos cilindros con un pulverizador ultrasónico respecto al carburador original y estudiaron el desempeño del motor a diferente velocidades y cargas. Ellos operaron el motor con relaciones aire-combustible de hasta 27:1 y observaron una disminución en las emisiones de monóxido de carbono y óxidos de nitrógeno, pero un aumento considerable en las emisiones de hidrocarburos.

El presente trabajo es una prueba de concepto sobre el uso de inyectores ultrasónicos como forma de mejorar significativamente el desempeño de los motores de gasolina. Las ventajas del inyector se comprobaron mediante la implementación de un inyector ultrasónico indirecto en un motor de motocicleta Honda 125. El desempeño se juzgó por las emisiones del inyector en comparación con el carburador original del motor. Finalmente, se analizaron los resultados obtenidos de las pruebas del motor.

\section{PULVERIZADOR ULTRASÓNICO}

Las observaciones experimentales han demostrado que la cavitación y las ondas capilares son los mecanismos causantes de la atomización ultrasónica. Adicionalmente, el fenómeno del viento de cuarzo se presenta en forma de una fuente en la superficie del líquido atomizado (Gallego y Graff, 2015).

Cuando se dirigen ondas ultrasónicas de alta intensidad a una interfaz líquido-gaseosa, aparecen ondas capilares en la superficie. Las oscilaciones crecen hasta que expelen partículas de sus crestas. La longitud de onda de las ondas capilares depende de la frecuencia de la onda acústica, pudiendo ser el tamaño de las partículas de la neblina uniforme y controlado, y adicionalmente llegar a valores micrométricos (Cavalieri et al., 2016).

Para construir el atomizador de combustible se seleccionó un transductor piezoeléctrico de PZT que opera a $1.7 \mathrm{MHz}$ y puede atomizar un gasto de gasolina de $10 \mathrm{ml} / \mathrm{min}$. El diámetro medio de la neblina atomizada, $d$, se evalúa con la correlación universal, Ecuación (1), propuesta por Rajan y Pandit (2001). En dicha ecuación $\rho$ es la densidad 
del líquido, $\sigma$ la tensión superficial, $f$ la frecuencia de la onda acústica, We el número de Webber, $O h$ el número de Ohnesorge e In el número de intensidad. En el cálculo se consideran las propiedades del transductor, Tabla 1 (PZT Electronic Ceramic Co., 2018), y de la gasolina Pemex Premium, Tabla 2 (Castillo, 2008). El diámetro medio de la neblina se calcula de 24 micras.

$d=\sqrt[3]{\frac{\pi \sigma}{\rho f^{2}}}\left(1+0.1 W e^{0.22} O h^{0.166} I^{-0.0277}\right.$

\section{Tabla 1: Propiedades del transductor}

\begin{tabular}{cc}
\hline Propiedad & Valor \\
\hline Frecuencia & $1.7 \mathrm{MHz}$ \\
Diámetro & $20 \mathrm{~mm}$ \\
Espesor & $1.2 \mathrm{~mm}$ \\
Capacitancia & $1.8 \mathrm{nF}$ \\
Voltaje & $<48 \mathrm{~V}$ \\
Gasto & $10 \mathrm{ml} / \mathrm{min}$ \\
Coeficiente de acoplamiento & $>52 \%$ \\
electromecánico & \\
\hline
\end{tabular}

Tabla 2: Propiedades de la gasolina Pemex Premium

\begin{tabular}{cc}
\hline Propiedad & Valor \\
\hline Densidad & $706 \mathrm{~kg} / \mathrm{m}^{3}$ \\
Tensión superficial & $22 \mathrm{mN} / \mathrm{m}$ \\
Viscosidad & $0.5 \mathrm{mPa} \cdot \mathrm{s}$ \\
Módulo de compresibilidad & $1.125 \mathrm{GPa}$ \\
\hline
\end{tabular}

El inyector consiste en una cámara de pulverización (Fig. 1-e), una trampa de separación (Fig. 1-d) y una boquilla que suministra la neblina al colector de admisión (Fig. 1-c).

La cámara de pulverización es un depósito de combustible en cuyo fondo se encuentra el transductor (Fig. 1-f). En la cámara se atomiza la gasolina y aparece la fuente consecuencia del viento de cuarzo (Fig. 1-g). Por los bordes de la cámara ingresa un flujo de aire que arrastra la neblina hacia la trampa de separación. La trampa consiste en un plato invertido que detiene la fuente y contiene las gotas que lanza. Alrededor de la trampa fluye la neblina hacia la boquilla, la cual inyecta al flujo en la entrada del colector de admisión. El flujo de aire es inducido por la succión del motor.
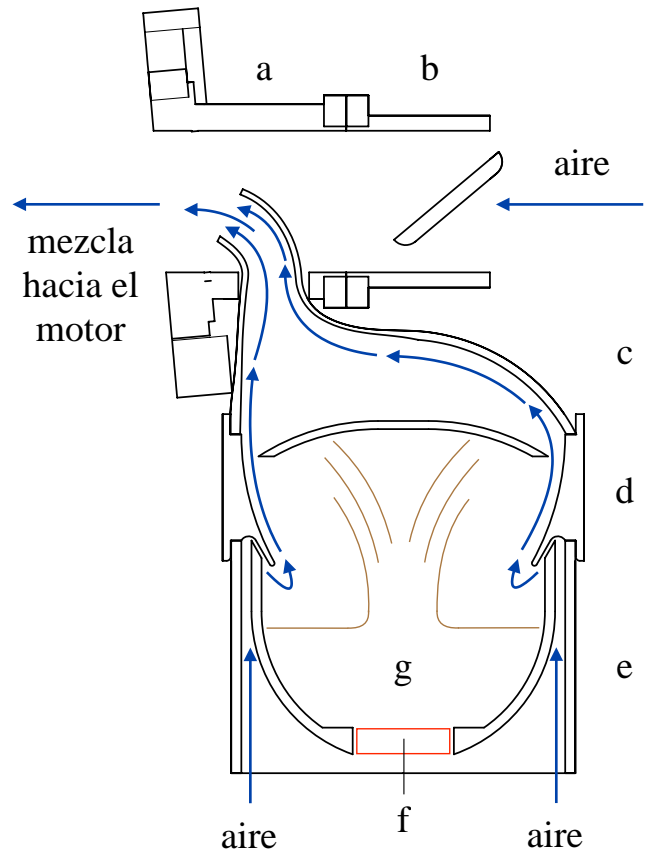

Fig. 1. Operación del inyector ultrasónico: colector de admisión (a) válvula mariposa (b) boquilla (c) trampa de separación (d) cámara de pulverización $(e)$ transductor $(f)$ gasolina $(g)$

Teniendo en cuenta que el inyector opera en contacto directo con gasolina y con el colector de admisión del motor-el cual tiene una temperatura próxima a $60{ }^{\circ} \mathrm{C}$ operando en caliente-se utilizó nylon para elaborar el prototipo empleando manufactura aditiva como proceso de fabricación.

\section{ARREGLO EXPERIMENTAL}

Las pruebas se realizaron en un motor de ignición por chispa de cuatro tiempos y un cilindro Honda CGL 125; sus especificaciones se enlistan en la Tabla 3 (Moto Honda da Amazônia Ltda., 2001).

Tabla 3: Especificaciones del motor de pruebas

\begin{tabular}{cc}
\hline Parámetro & Descripción \\
\hline Diámetro del pistón & $56.5 \mathrm{~mm}$ \\
Carrera & $49.5 \mathrm{~mm}$ \\
Cilindrada & $124.1 \mathrm{~cm}^{3}$ \\
Relación de compresión & $9: 1$ \\
Válvulas & OHV (válvulas en la culata) \\
Lubricación & Forzada a presión y baño en \\
Enfriamiento & aceite \\
Peso en seco & Forzado por aire \\
Encendido & $29.8 \mathrm{~kg}$ \\
& CDI (ignición por descarga \\
& de capacitor)
\end{tabular}


Las emisiones y la velocidad del motor se midieron utilizando un analizador de gases BrainBee AGS688 (Brain Bee, 2014). La temperatura del motor se midió en la superficie de la culata del motor con una cámara termográfica Fluke Ti400 (Fluke, 2013).

Dados los niveles de monóxido e hidrocarburos que emite el motor, se implementó un sistema de muestreo para tomar medidas representativas de las emisiones. El sistema consiste en un túnel de dilución de acero galvanizado (Fig. 2b). En un extremo del tubo se colocó un ventilador que induce el flujo de aire para la dilución (Fig. 2a), el otro extremo del tubo fungió como puerto de muestreo. El escape del motor se conectó al túnel de dilución (Fig. 2c).

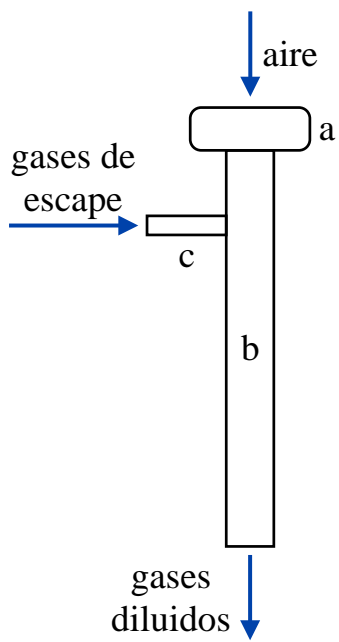

Fig. 2. Esquema del sistema de muestreo: (a) ventilador $(b)$ túnel de dilución (c) escape

En una prueba preliminar, con el inyector ultrasónico, el motor encendió tanto en frío $\left(30{ }^{\circ} \mathrm{C}\right.$ superficial en la culata) como en caliente $\left(100{ }^{\circ} \mathrm{C}\right.$ superficial en la culata). La velocidad a la que se obtuvo una combustión constante fue de 7000 rpm.

\subsection{Metodología}

Para comparar en condiciones estandarizadas el desempeño del motor equipado con los distintos sistemas de admisión, se estableció un protocolo basado en la norma mexicana vigente de verificación de motocicletas (SEMARNAT, 1993). La Fig. 3 muestra el arreglo utilizado para registrar las emisiones.

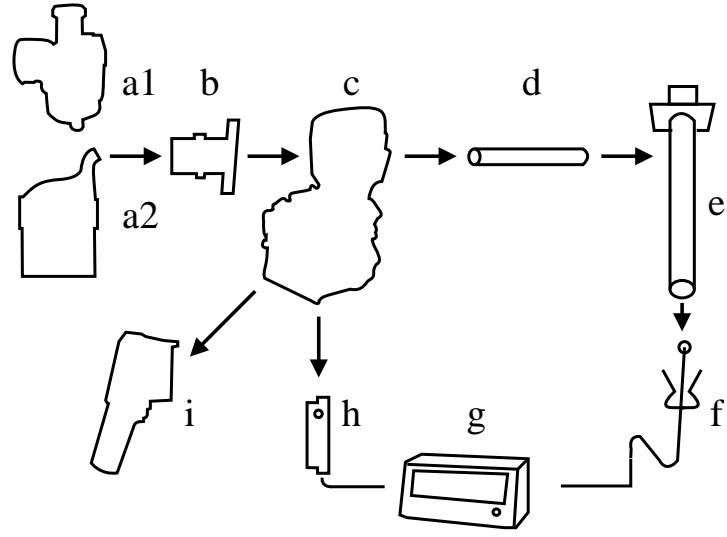

Fig. 3. Esquema del arreglo experimental: (a1) carburador (a2) inyector ultrasónico (b) colector de admisión (c) motor (d) escape (e) sistema de muestreo $(f)$ sonda de muestreo $(g)$ analizador de gases $(h)$ tacómetro $(i)$ cámara termográfica

Las pruebas fueron realizadas en ralentí y la velocidad se ajustó a $7000 \mathrm{rpm}$, pues fue la velocidad dónde el motor equipado con el inyector ultrasónico operó de manera más estable.

\subsubsection{Preparación del banco de pruebas}

1. Abrir el respiradero del cárter.

2. Calibrar el analizador de gases con aire atmosférico.

3. Conectar la sonda al puerto de muestreo (Fig. $3 f)$.

4. Conectar el tacómetro a la bujía (Fig. 3h).

\subsubsection{Ensayo de arranque en frío}

1. Partiendo de la temperatura ambiente, encender el motor, acelerarlo a $7000 \mathrm{rpm}$ y mantener esta velocidad $( \pm 1000 \mathrm{rpm})$.

2. Esperar 20 segundos para estabilizar las mediciones.

3. Registrar las lecturas durante 10 segundos.

\subsubsection{Ensayo de operación en caliente}

1. Encender el motor y dejarlo operar hasta que alcance $100{ }^{\circ} \mathrm{C}$.

2. Acelerar al motor a $7000 \mathrm{rpm}$ y mantener esta velocidad ( $\pm 1000 \mathrm{rpm})$.

3. Esperar 20 segundos para estabilizar las mediciones.

4. Registrar las lecturas durante 10 segundos. 


\section{ANÁLISIS}

Las mediciones obtenidas sirven para comparar relativamente los niveles de emisiones. Los valores no son absolutos debido al sistema de dilución, las emisiones reales son mayores. Como consecuencia de la dilución no se midió la relación estequiométrica de la combustión ni las emisiones de NOx.

\subsection{Resultados}

Tabla 4: Valores medios del ensayo de arranque en frío

\begin{tabular}{cccccc}
\hline Pulverizador & RPM & $\begin{array}{c}\text { O2 } \\
\text { Unidades }\end{array}$ & $\begin{array}{c}\text { CO2 } \\
\%\end{array}$ & $\begin{array}{c}\text { CO } \\
\%\end{array}$ & $\begin{array}{c}\text { HC } \\
\text { ppm }\end{array}$ \\
\hline Carburador & 7303 & 18.59 & 1.91 & 0.06 & 116 \\
$\begin{array}{c}\text { Inyector } \\
\text { ultrasónico }\end{array}$ & 7198 & 19.82 & 0.98 & 0.04 & 1436 \\
\hline
\end{tabular}

Tabla 5: Desviación estándar del ensayo de arranque en frío

\begin{tabular}{cccccc}
\hline $\begin{array}{c}\text { Pulverizador } \\
\text { Unidades }\end{array}$ & $\begin{array}{c}\text { RPM } \\
r p m\end{array}$ & $\begin{array}{c}\text { O2 } \\
\%\end{array}$ & $\begin{array}{c}\text { CO2 } \\
\%\end{array}$ & $\begin{array}{c}\text { CO } \\
\%\end{array}$ & $\begin{array}{c}\text { HC } \\
\text { ppm }\end{array}$ \\
\hline $\begin{array}{c}\text { Carburador } \\
\text { Inyector }\end{array}$ & 186 & 0.11 & 0.09 & 0.005 & 13 \\
ultrasónico & 541 & 0.06 & 0.06 & 0.004 & 65 \\
\hline
\end{tabular}

En el ensayo en frío, el inyector ultrasónico produjo un incremento en las emisiones de hidrocarburos y oxígeno, pero una disminución en las emisiones de monóxido y bióxido de carbono (Tabla 4). La velocidad media del motor equipado con el inyector fue menor a la del carburador, mas su desviación fue tres veces mayor (Tabla 5). Tal incremento junto con el aumento de oxígeno y la disminución de bióxido de carbono indican una menor cantidad de combustible quemado.

\section{Tabla 6: Valores medios del ensayo de operación en caliente}

\begin{tabular}{cccccc}
\hline $\begin{array}{c}\text { Pulverizador } \\
\text { Unidades }\end{array}$ & $\begin{array}{c}\text { RPM } \\
\text { rpm }\end{array}$ & $\begin{array}{c}\text { O2 } \\
\%\end{array}$ & $\begin{array}{c}\text { CO2 } \\
\%\end{array}$ & $\begin{array}{c}\text { CO } \\
\%\end{array}$ & $\begin{array}{c}\text { HC } \\
\text { Ppm }\end{array}$ \\
\hline Carburador & 7110 & 18.84 & 1.64 & 0.06 & 176 \\
$\begin{array}{c}\text { Inyector } \\
\text { ultrasónico }\end{array}$ & 7145 & 19.93 & 0.92 & 0.04 & 1505 \\
\hline
\end{tabular}

Tabla 7: Desviación estándar del ensayo de operación en caliente

\begin{tabular}{cccccc}
\hline $\begin{array}{c}\text { Pulverizador } \\
\text { Unidades }\end{array}$ & $\begin{array}{c}\text { RPM } \\
\mathrm{rpm}\end{array}$ & $\begin{array}{c}\text { O2 } \\
\%\end{array}$ & $\begin{array}{c}\text { CO2 } \\
\%\end{array}$ & $\begin{array}{c}\text { CO } \\
\%\end{array}$ & $\begin{array}{c}\text { HC } \\
\mathrm{ppm}\end{array}$ \\
\hline $\begin{array}{c}\text { Carburador } \\
\text { Inyector }\end{array}$ & 279 & 0.26 & 0.20 & 0.003 & 22 \\
ultrasónico & 457 & 0.07 & 0.09 & 0.000 & 45 \\
\hline
\end{tabular}

En el ensayo en caliente el motor mostró un incremento en las emisiones de hidrocarburos para ambos pulverizadores (Tabla 6). No obstante, se observó una disminución en la producción de monóxido y bióxido de carbono. La desviación de la velocidad del motor equipado con el carburador aumentó respecto del ensayo en frío, pero la del inyector disminuyó (Tablas 5 y 7). La velocidad media alcanzada con el inyector superó a la del carburador en la prueba en caliente, no obstante, la desviación fue 1.6 veces mayor a la producida por el carburador.

\subsection{Discusión}

La elevada presencia de hidrocarburos no quemados y el aumento en el oxígeno de la muestra indican fallos en la ignición (misfiring). Tal fenómeno provocó que el motor expulsara, en algunos ciclos, la mezcla de aire-combustible sin quemar. Tal inconsistencia en la combustión se corroboró con la mayor desviación en la velocidad del motor equipado con el inyector, pues la combustión no ocurrió de forma continua.

El motor equipado con el carburador disminuyó sus emisiones de hidrocarburos durante la operación en caliente; mientras que el motor equipado con el inyector, en frío o en caliente, emitió las mismas cantidades. Tal fenómeno, igualmente corresponde con la presencia de fallos en la ignición, pues las emisiones de hidrocarburos del inyector no están ligadas a la temperatura de operación del motor durante la combustión.

Considerando que las emisiones de bióxido de carbono son proporcionales al combustible quemado por el motor, el inyector ultrasónico permitió al motor operar, en promedio, con 38\% menos combustible del que requirió para el carburador. A pesar de ello, el motor demandó una mayor cantidad de combustible que no quemó por los fallos en la ignición.

Al poder generar una neblina más fina de combustible se tiene una combustión más completa, lo que reduce las emisiones de monóxido de carbono y de hidrocarburos; así como disminuye el consumo de combustible y aumenta la temperatura de operación del motor. Dicho comportamiento fue obtenido por Kumar et al. (2013) y también por Namiyama et al. (1989).

Un comportamiento similar al observado-los hidrocarburos se elevaron, pero bajaron las demás emisiones contaminantes y el CO-fue obtenido por 
Balasubramanian et al. (2015) quiénes igualmente utilizaron un transductor sumergido en combustible como atomizador, a una frecuencia de $2.4 \mathrm{MHz}$. Kumar et al. y Balasubramanian et al. destacan la importancia del control del ingreso de aire para evitar fallos en la ignición, causados por una relación aire-combustible excesivamente alta. Kumar et al. controloaron tal fenómeno y reportaron que el atomizador ultrasónico permitió el funcionamiento a relaciones aire-combustible altas, pero a partir de 21 ocurrieron fallos en la ignición y, como consecuencia, los hidrocarburos se elevaron. Balasubramanian et al. realizaron sus experimentos a relaciones aire-combustible de 20 a 27 , lo cual los llevó a observar grandes cantidades de hidrocarburos no quemados a la salida del motor.

Las emisiones medidas y el comportamiento no constante observado del motor equipado con el inyector ultrasónico demuestran que se trabajó con una relación aire-combustible ligera que no permitió la combustión constante de la mezcla suministrada. Dado lo reportado por Balasubramanian y Kumar, la relación a la que operó el motor fue superior a 21.

\section{CONCLUSIONES}

Se desarrolló un inyector de combustible que pulveriza gasolina mediante vibraciones ultrasónicas para obtener una neblina de combustible con un diámetro medio de 24 micras. $\mathrm{El}$ inyector permitió arrancar y operar un motor de un cilindro y cuatro tiempos.

Los valores medidos mostraron una disminución en el monóxido de carbono de $67 \%$ tanto para el arranque en frío del motor como para la operación en caliente. Una disminución del $50 \%$ en las emisiones de $\mathrm{CO} 2$ indican un menor consumo de combustible por parte del motor. Las emisiones de hidrocarburos presentaron un aumento significativo (1237\% durante el arranque en frío y $855 \%$ durante la operación en caliente) las cuales indican fallas en la ignición: no todo el combustible se quema y se expulsa como hidrocarburos en los gases de escape. La presencia de fallas en la ignición corresponde con la mayor desviación observada en la velocidad del motor: 3 veces superior durante el arranque en frío y 1.64 veces superior durante la operación en caliente.

\subsection{Trabajo a futuro}

Es necesario modificar al inyector para obtener una combustión ininterrumpida del motor que permita controlar su velocidad y disminuir las emisiones de hidrocarburos; así como modificar la instrumentación para poder caracterizar el desempeño del inyector y compararlo con sistemas comerciales (López et al., 2010), adicionalmente debemos considerar un tratamiento adecuado de la incertidumbre relacionada con las mediciones (Gan, 2008):

- Controlar la relación aire combustible de la mezcla.

- Medir el consumo de combustible del inyector.

- Medir la temperatura de operación y tiempo de calentamiento del motor.

- Medir los valores reales de las emisiones.

- Caracterizar la dispersión del tamaño de partículas mediante el diámetro medio de Sauter de la neblina atomizada.

- Realizar ensayos a diferentes velocidades y cargas.

- Implementar el sistema en un vehículo comercial.

\section{RECONOCIMIENTO}

A la Universidad Nacional Autónoma de México, al proyecto PAPIME PE108418 "Desarrollo de equipo y manuales para prácticas de laboratorio de motores de combustión interna" por la beca recibida que permitió el satisfactorio desarrollo del presente proyecto.

Al proyecto PAPIIT IG101018 "Simulación de la combustión en motores de combustión interna usando biocombustibles, y medición de la masa de contaminantes, a fin de estructurar un inventario de emisiones en el Valle de México" por la ayuda proporcionada para la compra de material.

A la Lic. Josefina Elizabeth Plata García de Unidad de Servicios de Información del Instituto de Ingeniería de la UNAM por su apoyo en la búsqueda de información bibliográfica.

Al Dr. Fernando Pérez Escamirosa del Departamento de Cirugía de la Facultad de Medicina de la UNAM, por el apoyo otorgado en la manufactura del prototipo.

\section{REFERENCIAS}

Balasubramanian, N., Keerthi, G. y Anand, T. N. C. (2015). "Fuelling a Two-Wheeler Engine 
by Ultrasonic Atomization". Small Engine Technologies Conference \& Exhibition, 201532-0757.

Brain Bee (2014). AGS-688 Gas Analyser 2 or 4 stroke petrol engines User manual, Brain Bee S.p.A., Parma.

BP (2018). BP Energy Outlook 2018 Edition, BP Energy Economics, Londres.

Castillo H., P. (2008). "Estudio de las propiedades fisicoquímicas de gasolina y diesel mexicanos con etanol al $10 \%$ y $15 \%$ en volumen". Tesis de maestría, Instituto Tecnológico y de Estudios Superiores de Monterrey.

Cavalieri, F., Chemat, F., Okitsu, K., Sambandam, A., Yasui, K. y Zisu, B. (2016). Handbook of Ultrasonics and Sonochemistry, Springer, Singapore.

Fluke (2013). Ti200, Ti300, Ti400 Thermal Imagers Users Manual, Fluke Corporation, Everett.

Gallego J., J. A. y Graff, K. F. (2015). Power Ultrasonics, Woodhead Publishing, Sawston.

Gan A., A. (2008). "El concepto de medición en ingeniería”. Revista Colombiana de Tecnologías de Avanzada, Vol. 1, No. 11.

International Energy Agency (2017). World Energy Balances 2017: Overview, OECD Publishing, Paris.

Kumar R., S., Ajay, M. y Anand, T. N. C. (2013). "Studies on a 4-Stroke Spark Ignition Engine with an Ultrasonic Atomizer". 23rd National Conference on I. C. Engine and Combustion, DOI: $10.13140 / 2.1 .3421 .0881$.

López P., J. L., Tarantino A., R. y Aranguren Z., S. (2010). "Metodología para la selección de los instrumentos que mayor impacto de fallas presentan en el generador de vapor de la empresa Termotasajero Colgener". Revista Colombiana de Tecnologías de Avanzada, Vol. 1, No. 15.

Namiyama, K., Nakamura, H., Kokubo, K. y Hosogai, D. (1989). "Development of Ultrasonic Atomizer and Its Application to S.I. Engines”. SAE Technical Paper, 890430.

Rajan, R. y Pandit, A. C. (2001). "Correlations to Predict Droplet Size in Ultrasonic Atomisation”. Ultrasonics, Vol. 39, No. 4.

SEMARNAT. (1993). Norma oficial mexicana, que establece las caracteristicas del equipo y el procedimiento de medicion, para la verificacion de los niveles de emision de gases contaminantes, provenientes de las motocicletas en circulacion que usan gasolina o mezcla de gasolina-aceite como combustible, México.

Tanyeri, B. y Öner, C. (2014). "The Investigation of Using For Gasoline Atomization of Ultrasonic Sound Generated By Piezoelectric Ceramic". Periodicals of Engineering and Natural Sciences, Vol. 2, No. 1.

\section{SITIOS WEB}

Furu, L. (2012). Car running on ultrasonic gasoline mist, YouTube.

https://www.youtube.com/watch?v=n150d9DGtw

$\mathrm{w}$

(Consultado: 14 de junio 2017)

PZT Electronic Ceramic Co. (2018). Mist Generation Transducer, Piezoelements, China. http://www.piezoelements.com/mistgeneration/piezo-transducer-atomizer/mistgeneration-transducer.html (Consultado: 6 de febrero 2018)

Winton, N. (2016). Global EV, hybrid sales to soar, with U.S. reluctance, The Detroit News, Estados Unidos.

http://www.detroitnews.com/story/business/column ists/neil- winton/2016/11/25/winton-alternativefuel-car-sales-estimates/94426704

(Consultado: 7 de agosto 2017) 